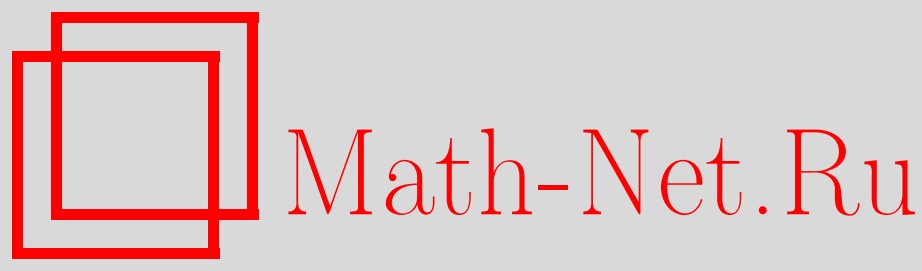

Н. Н. Боголюбов (мл.), А. К. Прикарпатский, У. Танери, Структура вакуума, специальная теория относительности и квантовая механика: возврат к теоретикополевому безгеометрическому подходу, ТМФ, 2009, том 160, номер 2, 249-269

DOI: https://doi.org/10.4213/tmf6396

Использование Общероссийского математического портала Math-Net.Ru подразумевает, что вы прочитали и согласны с пользовательским соглашением http://www . mathnet.ru/rus/agreement

Параметры загрузки:

IP : 35.173 .219 .149

26 апреля 2023 г., 03:41:17

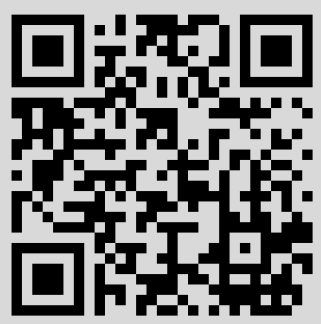




\title{
ФИЗИКА
}

Том 160, № 2

август, 2009

2009 г. Н. Н. Боголюбов (мл.)*, А. К. Прикарпатский ${ }^{\dagger}$, У. Танери ${ }^{\ddagger}$

\section{СТРУКТУРА ВАКУУМА, СПЕЦИАЛЬНАЯ ТЕОРИЯ ОТНОСИТЕЛЬНОСТИ И КВАНТОВАЯ МЕХАНИКА: ВОЗВРАТ К ТЕОРЕТИКО-ПОЛЕВОМУ БЕЗГЕОМЕТРИЧЕСКОМУ ПОДХОДУ}

\begin{abstract}
Сформулированы основные фундаментальные принципы, характеризующие полевую структуру вакуума, а также проанализированы модель ассоциированной вакуумной среды и динамика заряженной точечной частицы с помощью развитых теоретико-полевых методов. Рассмотрен новый подход к электродинамической теории Максвелла, вновь выведены основные уравнения этой теории на основе предложенных принципов полевой структуры вакуума, получено классическое соотношение специальной теории относительности между энергией и соответствующей массой точечной частицы. Вновь рассмотрено и проанализировано выражение для силы Лоренца в произвольных неинерциальных системах отсчета, а также представлены новые интерпретации соотношений между специальной теорией относительности и квантовой механикой. Получены квантово-механические уравнения типа Шредингера для релятивистской точечной частицы во внешних потенциальных и магнитных полях в пределе квазиклассической аппроксимации, когда постоянная Планка $\hbar \rightarrow 0$ и скорость света $c \rightarrow \infty$.
\end{abstract}

Ключевые слова: структура вакуума, закон сохранения локальной массы и импульса, сила Лоренца, теория относительности.

*Математический институт им. В. А. Стеклова РАН, Москва, Россия; The Abdus Salam International Centre for Theoretical Physics, Trieste, Italy.

E-mail: nikolai_bogolubov@hotmail.com

${ }^{\dagger}$ The AGH University of Science and Technology, Department of Applied Mathematics, Krakow, Poland; Дрогобычский государственный педагогический университет им. И. Франко, г. Дрогобыч, Украина. E-mail: pryk.anat@ua.fm, prykanat@cybergal.com

${ }^{\ddagger}$ Eastern Mediterranean University, Department of Applied Mathematics and Computer Science, Famagusta; Kyrenia American University, Institute of Graduate Studies, Kyrenia, Cyprus. E-mail: ufuk.taneri@gmail.com 
Посвящается академику Николаю Николаевичу Боголюбову в честь столетия со дня его рождения с искренней признательностью к его блестящему таланту и впечатляющему вкладу в современную нелинейную математику и квантовую физику

\section{1. ВВЕДЕНИЕ}

Считается общепринятым утверждение, что ни одна физическая теория не может представлять абсолютно верную картину Природы, поскольку всегда существуют границы ее применимости, которые определяются экспериментами и новыми опытными данными. Это утверждение касается, очевидно, и теории относительности, которая по замыслу Эйнштейна должна была логически возникнуть как из принципа постоянства скорости света по отношению к инерциальным системам отсчета, так и из обобщенного принципа эквивалентности по отношению к гравитации и инерциальным массам. Эта теория, а именно специальная теория относительности (CTO), была подтверждена многими экспериментами в области ядерной физики, которые в то же время явились началом ядерной энергетики, широко используемой в мире.

Однако природа пространства-времени и окружающих объектов была и остается одной из наиболее интересных проблем, стоящих перед наукой и, в частности, перед естествоиспытателями. Одним из наиболее ярких открытий в физике XIX века было объединение понятий электричества и магнетизма на основе теории электромагнетизма Фарадея-Максвелла. Эта теория объяснила главные физические законы распространения света в пространстве-времени и выдвинула новые вопросы, касающиеся природы вакуума. Тем не менее все попытки выяснить природу вакуума оказались безуспешными, несмотря на новые идеи, предложенные Махом, Лоренцем, Пуанкаре, Эйнштейном и некоторыми другими физиками. Более того, необычный подход к понятию пространства-времени, предложенный Эйнштейном, не способствовал решению проблем, связанных с природой физического вакуума [1]-[7], сводя их к некоторым физически немотивированным формальным математическим принципам и рецептам, объединенным в СТО, которая оказалась адаптированной только к инерциальным системам отсчета и встретилась с большими проблемами при объяснении электромагнитных сил Лоренца и соотношений между инерциальными и гравитационными силами. Эти проблемы были искусственно разрешены с помощью принципа эквивалентности, в котором постулируется совпадение инерциальной массы материального объекта с его гравитационной массой. В противоположность этому Мах предположил, что любое движение материальной точки в пространстве-времени, как прямолинейное, так и криволинейное, также может быть относительным. В частности, так как закон инерции также является относительным, мера инерции материальной точки, т.е. ее масса, должна также быть относительной и зависеть от взаимодействий между всеми материальными телами во Вселенной. Эти идеи Маха сильно повлияли на Эйнштейна, и он пытался согласовать принцип относительности Маха со своим собственным подходом к общей 
теории относительности. Тем не менее, несмотря на титанические усилия Эйнштейна, ему не удалось включить принцип относительности Маха в свою общую теорию относительности, так как они оказались взаимоисключающими.

Одновременно сущность вакуума как физического явления почти полностью исчезла из теории Эйнштейна, будучи замещенной геометризацией природы пространства-времени и, соответственно, всех физических явлений. Тем временем поразительные успехи квантовой физики XX века, особенно квантовой электродинамики, продемонстрировали достаточно ясно [6], [8]-[14], что поляризация вакуума и явления электрон-позитронной аннигиляции приводят к новым вопросам о структурах пространства-времени и вакуума и дают возможность переоценки [3], [4], [7], [15]-[20] существующих точек зрения на них.

Как хорошо известно, классическая механика использует понятие потенциальной энергии, являющейся скалярной функцией пространственных переменных, очень важной для формулирования динамических уравнений, несмотря на тот факт, что она определяется с точностью до произвольной постоянной. Подобная ситуация наблюдается в классической электродинамической теории, которая эффективно использует понятие скалярных и векторных потенциалов, связанных между собой при помощи хорошо известного калибровочного условия совместимости Лоренца [9]-[11], [21] и определенного с точностью до соответствующих калибровочных преобразований.

Эти, в некотором смысле разные, потенциальные функции были позже глубоко проанализированы в рамках классической теории относительности Эйнштейна многими физиками [4], [10], [14]-[26], что привело к пониманию их фундаментальной роли в объединении двух теорий: электромагнетизма и гравитации. Более того, вследствие знаменитого соотношения Эйнштейна между внутренней энергией и массой, зависящей от скорости материальной частицы, возникли новые проблемы. Однако, как было отмечено в монографии [15], соотношение между массой частицы и ее внутренней энергией учитывает только кинетическую энергию, не затрагивая массы, связанной с потенциальной энергией. Это делает классическую теорию относительности не вполне замкнутой и физически несогласованной теоретической конструкцией. Бриджмен в монографии [27] писал: “Эйнштейн не перенес в свою общую теорию относительности те уроки и прозрения, с которыми сам же знакомил нас в своей специальной теории".

Мы рассмотрим некоторые нетривиальные аспекты проблем реального пространства-времени, глубоко связанных с теорией относительности и электродинамикой, с целью вывода из естественных теоретико-полевых принципов всех результатов теории электромагнетизма Максвелла и теории относительности, показывая их относительное или только видимое совпадение с реальными физическими явлениями и подчеркивая новые перспективы, стоящие перед современной фундаментальной физикой.

Более того, применяя далее полевой подход к микроскопической структуре вакуума, предложенный в работе [17] и описанный в работе [7], мы получим в пределе квазиклассической аппроксимации новый вывод основных квантово-механических соотношений, описывающих эволюцию микроскопических систем частиц, совпадающих при $\hbar \rightarrow 0$ с результатами, полученными в начале $\mathrm{XX}$ века великими физиками Шредингером, Гейзенбергом и Дираком. 


\section{2. ЭЛЕКТРОМАГНИТНАЯ ТЕОРИЯ МАКСВЕЛЛА: НОВЫЙ ВЗГЛЯД И ИНТЕРПРЕТАЦИЯ}

Рассмотрим теоретико-полевую модель [7] микроскопической структуры вакуума как некоторую физическую реальность, погруженную в стандартную трехмерную евклидову систему отсчета $\mathbb{E}^{3}:=\left(\mathbb{R}^{3},\langle\cdot, \cdot\rangle\right)$, отмеченную тремя пространственными координатами $r \in \mathbb{E}^{3}$, наделенную скалярным произведением $\langle\cdot, \cdot\rangle$ и параметризованную при помощи скалярного временно́го параметра $t \in \mathbb{R}$. Мы будем описывать физическую вакуумную среду, наделяя ее достаточно гладкой четырехкомпонентной векторной потенциальной функцией $(W, A): \mathbb{E}^{3} \times \mathbb{R} \rightarrow \mathbb{R} \times \mathbb{E}^{3}$, естественно связанной со свойствами распространения света. Материальные объекты, погруженные в вакуум, мы будем моделировать (классически) при помощи скалярной функции плотности электрического заряда $\rho: \mathbb{E}^{3} \times \mathbb{R} \rightarrow \mathbb{R}$ и векторной функции плотности тока $J: \mathbb{E}^{3} \times \mathbb{R} \rightarrow \mathbb{E}^{3}$, являющихся достаточно гладкими функциями. Воспользуемся следующими теоретико-полевыми принципами.

1. Первый теоретико-полевой принцип, который мы принимаем по отношению к вакууму, формулируется следующим образом: четырехвекторная функция $(W, A)$ : $\mathbb{E}^{3} \times \mathbb{R} \rightarrow \mathbb{R} \times \mathbb{E}^{3}$ удовлетворяет стандартному условию непрерывности типа Лоренца

$$
\frac{1}{c} \frac{\partial W}{\partial t}+\langle\nabla, A\rangle=0,
$$

где $\nabla:=\partial / \partial r-$ оператор градиента.

2. Второй теоретико-полевой принцип, который мы принимаем, есть динамическое условие на скалярный потенциал $W: \mathbb{E}^{3} \times \mathbb{R} \rightarrow \mathbb{R}:$

$$
\frac{1}{c^{2}} \frac{\partial^{2} W}{\partial t^{2}}-\nabla^{2} W=\rho .
$$

При этом мы предполагаем, что действует линейный закон распространения малых однородных и изотропных возмущений вакуумной среды в пространстве-времени, понимаемых здесь как первая (линейная) аппроксимация в случае достаточно слабых полей.

3. Третий принцип является простым условием согласованности первых двух, а именно стандартным условием непрерывности для функций плотности заряда и тока

$$
\frac{\partial \rho}{\partial t}+\langle\nabla, J\rangle=0 .
$$

Поскольку мы пытаемся вывести из первых принципов хорошо известные уравнения Максвелла для электромагнитного поля и проанализировать соответствующие силы Лоренца и соответствующие результаты специальной теории относительности, будем считать, что используемый выше параметр $c>0$ для вакуумных полевых возмущений совпадает со скоростью света в вакууме. Объединив уравнения (1) и (2), получаем

$$
\frac{1}{c^{2}} \frac{\partial^{2} W}{\partial t^{2}}=-\left\langle\nabla, \frac{1}{c} \frac{\partial A}{\partial t}\right\rangle=\langle\nabla, \nabla W\rangle+\rho,
$$


откуда

$$
\left\langle\nabla,-\frac{1}{c} \frac{\partial A}{\partial t}-\nabla W\right\rangle=\rho .
$$

Положив

$$
E:=-\frac{1}{c} \frac{\partial A}{\partial t}-\nabla W
$$

мы получаем первое материальное уравнение Максвелла

$$
\langle\nabla, E\rangle=\rho
$$

для электрического поля $E: \mathbb{E}^{3} \times \mathbb{R} \rightarrow \mathbb{E}^{3}$. Применив теперь операцию ротора $\nabla$ к выражению (5), мы получаем первое полевое уравнение Максвелла

$$
\frac{1}{c} \frac{\partial B}{\partial t}-\nabla \times E=0
$$

для магнитной полевой вектор-функции $B: \mathbb{E}^{3} \times \mathbb{R} \rightarrow \mathbb{E}^{3}$, определенной как

$$
B:=\nabla \times A .
$$

Чтобы вывести второе полевое уравнение Максвелла, воспользуемся (8), (1) и (5):

$$
\begin{aligned}
\nabla \times B & =\nabla \times(\nabla \times A)=\nabla\langle\nabla, A\rangle-\nabla^{2} A= \\
& =\nabla\left(-\frac{1}{c} \frac{\partial W}{\partial t}\right)-\nabla^{2} A=\frac{1}{c} \frac{\partial}{\partial t}\left(-\nabla W-\frac{1}{c} \frac{\partial A}{\partial t}+\frac{1}{c} \frac{\partial A}{\partial t}\right)-\nabla^{2} A= \\
& =\frac{1}{c} \frac{\partial E}{\partial t}+\left(\frac{1}{c^{2}} \frac{\partial^{2} A}{\partial t^{2}}-\nabla^{2} A\right) .
\end{aligned}
$$

С учетом (3), (5), (6) имеем

$$
\left\langle\nabla, \frac{1}{c} \frac{\partial E}{\partial t}\right\rangle=\frac{1}{c} \frac{\partial \rho}{\partial t}=-\frac{1}{c}\langle\nabla, J\rangle,
$$

или

$$
\left\langle\nabla,-\frac{1}{c^{2}} \frac{\partial^{2} A}{\partial t^{2}}-\nabla\left(\frac{1}{c} \frac{\partial W}{\partial t}\right)+\frac{1}{c} J\right\rangle=0 .
$$

Теперь, используя (1), из (10) получаем

$$
\begin{aligned}
\left\langle\nabla,-\frac{1}{c^{2}} \frac{\partial^{2} A}{\partial t^{2}}-\nabla\left(\frac{1}{c} \frac{\partial W}{\partial t}\right)+\frac{1}{c} J\right\rangle & =\left\langle\nabla,-\frac{1}{c^{2}} \frac{\partial^{2} A}{\partial t^{2}}+\nabla\langle\nabla, A\rangle+\frac{1}{c} J\right\rangle= \\
& =\left\langle\nabla,-\frac{1}{c^{2}} \frac{\partial^{2} A}{\partial t^{2}}+\nabla^{2} A+\nabla \times(\nabla \times A)+\frac{1}{c} J\right\rangle= \\
& =\left\langle\nabla,-\frac{1}{c^{2}} \frac{\partial^{2} A}{\partial t^{2}}+\nabla^{2} A+\frac{1}{c} J\right\rangle=0 .
\end{aligned}
$$

Тем самым уравнение (11) дает

$$
\frac{1}{c^{2}} \frac{\partial^{2} A}{\partial t^{2}}-\nabla^{2} A=\frac{1}{c}(J+\nabla \times S)
$$


для некоторой гладкой вектор-функции $S: \mathbb{E}^{3} \times \mathbb{R} \rightarrow \mathbb{E}^{3}$. Необходимо отметить здесь, что уравнение непрерывности (3) определяет вектор плотности тока $J: \mathbb{E}^{3} \times \mathbb{R} \rightarrow \mathbb{E}^{3}$ с точностью до вихревого выражения, т.е. $J \simeq J+\nabla \times S$ и уравнение (12) может быть переписано в виде

$$
\frac{1}{c^{2}} \frac{\partial^{2} A}{\partial t^{2}}-\nabla^{2} A=\frac{1}{c} J
$$

Подставив (13) в (9), мы получаем второе полевое уравнение Максвелла

$$
\nabla \times B-\frac{1}{c} \frac{\partial E}{\partial t}=\frac{1}{c} J
$$

Из (8) также находится соотношение

$$
\langle\nabla, B\rangle=0 \text {. }
$$

Таким образом, мы вывели все уравнения электромагнитного поля Максвелла из наших трех основных принципов (1)-(3). Применим полученные результаты к результатам, следующим из теории относительности для случая точечных зарядов или масс. Ниже мы продемонстрируем соответствующие выводы, основанные на некоторых совершенно новых физических концепциях вакуумной среды, ранее описанных в работах [7], [17].

ЗАмЕчАниЕ. Проанализируем частный случай первого теоретико-полевого принципа (1) для вакуума, когда имеет место следующий локальный закон сохранения скалярной потенциальной функции $W: \mathbb{E}^{3} \times \mathbb{R} \rightarrow \mathbb{R}$ :

$$
\frac{d}{d t} \int_{\Omega_{t}} W d^{3} r=0,
$$

где $\Omega_{t} \subset \mathbb{E}^{3}$ - произвольная открытая область в пространстве $\mathbb{E}^{3}$ с гладкой границей $\partial \Omega_{t}$ для всех $t \in \mathbb{R}, d^{3} r$ - стандартная мера объема в $\mathbb{E}^{3}$ в окрестности точки $r \in \Omega_{t}$.

Вычислив выражение (16), получаем следующее эквивалентное (1) уравнение непрерывности:

$$
\frac{1}{c} \frac{\partial W}{\partial t}+\left\langle\nabla, \frac{v}{c} W\right\rangle=0,
$$

где $v:=d r / d t$ - вектор скорости возмущения вакуумной среды в точке $r \in \mathbb{E}^{3}$, ассоциированный с потенциальной полевой величиной $W$. Сравнивая уравнения (1), (17) и учитывая условие (3), получаем следующие соотношения:

$$
A=\frac{v}{c} W, \quad J=\rho v,
$$

хорошо известные в классической электродинамике и теории сверхпроводимости [2], [8]. Таким образом, мы пришли к новой физической интерпретации теории консервативного электромагнитного поля, когда векторный потенциал $A: \mathbb{E}^{3} \times \mathbb{R} \rightarrow \mathbb{E}^{3}$ полностью определен с помощью выражений (18) скалярной потенциальной функцией $W: \mathbb{E}^{3} \times \mathbb{R} \rightarrow \mathbb{R}$. Очевидно, что все уравнения электромагнитного поля Максвелла, выведенные выше, имеют место также и в случае (18), как это было показано в [17] (но с некоторыми математическими неточностями) и в работе [7]. 
Рассмотрим теперь уравнение сохранения (16) совместно с ассоциированным интегральным соотношением сохранения вакуумного "импульса"

$$
\frac{d}{d t} \int_{\Omega_{t}}\left(\frac{W v}{c^{2}}\right) d^{3} r=0,\left.\quad \Omega_{t}\right|_{t=0}=\Omega_{0}
$$

где $\Omega_{t} \subset \mathbb{E}^{3}$ - открытая область с гладкой границей $\partial \Omega_{t}$ для любого момента времени $t \in \mathbb{R}$, эволюция которой подчиняется уравнению

$$
\frac{d r}{d t}=v(r, t)
$$

для всех $x \in \Omega_{t}, t \in \mathbb{R}$, и зависит от начального состояния границы $\partial \Omega_{0}$. Из (19) получаем новое уравнение непрерывности

$$
\frac{d(v W)}{d t}+v W\langle\nabla, v\rangle=0
$$

Теперь, используя (17) в эквивалентной форме

$$
\frac{d W}{d t}+W\langle\nabla, v\rangle=0
$$

получаем локальное соотношение сохранения

$$
\frac{d v}{d t}=0
$$

скорости $v=d r / d t$ возмущений вакуумной среды, которое имеет место для всех значений параметра времени $t \in \mathbb{R}$. Как легко заметить, полученное соотношение полностью совпадает с хорошо известным гидродинамическим уравнением [28] идеальной сжимаемой жидкости без внешнего воздействия, т.е. любые внешние силы и полевое “давление" равны тождественно нулю. Мы получили вполне естественный результат, когда скорость распространения вакуумной полевой среды есть постоянная величина, равная скорости света в вакууме, если вспомнить исходное волновое уравнение (2), благодаря которому малые возмущения вакуумной полевой среды распространяются в пространстве со скоростью света.

\section{3. СПЕЦИАЛЬНАЯ ТЕОРИЯ ОТНОСИТЕЛЬНОСТИ И ДИНАМИЧЕСКИЕ УРАВНЕНИЯ ПОЛЯ}

Из классической электродинамики нам известно, что основное динамическое соотношение связывает ускорение массы частицы с силой Лоренца, которая существенно зависит от скорости заряда. Чтобы электродинамика была независимой от системы отсчета, физикам [8], [21] пришлось искусственно заменить преобразования Галилея преобразованиями Лоренца. Результатом этого позже стала теория относительности Эйнштейна, которая частично решила проблемы, возникшие с выводом "правильных" динамических уравнений для заряженной точечной частицы. Рассмотрим скалярную полевую функцию вакуумной среды $W: \mathbb{E}^{3} \times \mathbb{R} \rightarrow \mathbb{R}$ при 
условии консервативности (16), проанализированном выше. Это означает, что полевой векторный потенциал вакуумной среды $A: \mathbb{E}^{3} \times \mathbb{R} \rightarrow \mathbb{E}^{3}$, плотности заряда и тока $(\rho, J): \mathbb{E}^{3} \times \mathbb{R} \rightarrow \mathbb{R} \times \mathbb{E}^{3}$ связаны соотношением (18).

Рассмотрим теперь уравнения сохранения вакуумной полевой среды (17) и (2) для плотности $\rho=0$ :

$$
\begin{aligned}
-\frac{1}{c^{2}} \frac{\partial^{2} W}{\partial t^{2}} & =\frac{1}{c^{2}} \frac{\partial}{\partial t}\left(-\frac{\partial W}{\partial t}\right)=\frac{1}{c^{2}} \frac{\partial}{\partial t}(\langle\nabla, v W\rangle)= \\
& =\left\langle\nabla, \frac{\partial}{\partial t}\left(\frac{W v}{c^{2}}\right)\right\rangle=-\langle\nabla, \nabla W\rangle .
\end{aligned}
$$

Из соотношения (23) следует, что

$$
\frac{\partial}{\partial t}\left(\frac{W v}{c^{2}}\right)+\nabla W=\nabla \times F
$$

где $F: \mathbb{E}^{3} \times \mathbb{R} \rightarrow \mathbb{E}^{3}$ - некоторая гладкая функция, которую мы будем считать равной нулю согласно предполагаемой безвихревой динамике вакуумной среды. Кроме того, выражение (24), как было установлено выше (см. (5)), совпадает с внутривакуумным электрическим полем $E$, которое вследствие физической электронейтральности вакуумной среды должно, очевидно, равняться нулю, так как это поле согласно исходному полевому уравнению (2) пропорционально ее зарядовой плотности. Итак, наше динамическое уравнение на скалярную полевую функцию вакуумной среды $W: \mathbb{E}^{3} \times \mathbb{R} \rightarrow \mathbb{R}$ выглядит следующим образом:

$$
\frac{\partial}{\partial t}\left(\frac{W v}{c^{2}}\right)+\nabla W=0
$$

Рассмотрим точечную заряженную частицу $q$ в точке пространства $r=R(t):=$ $R_{0}+\int_{0}^{t} u(t) d t \in \mathbb{E}^{3}$, зависящей от параметра времени $t \in \mathbb{R}$ и начальной точки $R_{0} \in \mathbb{E}^{3}$ при $t=0$. Поле вакуумной среды описывается при помощи потенциальной полевой функции $W: \mathbb{E}^{3} \times \mathbb{R} \rightarrow \mathbb{R}$, которая естественным образом возмущена заряженной частицей $q$. Промоделируем это приближенно следующим результирующим функциональным соотношением:

$$
W(r, t)=\widetilde{W}(r, R(t))
$$

для некоторой скалярной функции $\widetilde{W}: \mathbb{E}^{3} \times \mathbb{E}^{3} \rightarrow \mathbb{R}$, которая должна удовлетворять (17), т.е.

$$
\left\langle\frac{\partial \widetilde{W}}{\partial R}, u\right\rangle+\langle\nabla, \widetilde{W} v\rangle=0
$$


Поскольку нас интересуют дифференциальные свойства функции $\widetilde{W}: \mathbb{E}^{3} \times \mathbb{E}^{3} \rightarrow \mathbb{R}$ при $r \rightarrow R(t) \in \mathbb{E}^{3}$, где локализована точечная заряженная частица, из (27) мы получаем соотношение

$$
\left.\left\langle\frac{\partial \widetilde{W}}{\partial R}+\frac{\partial \widetilde{W}}{\partial r}, u\right\rangle\right|_{r \rightarrow R(t)}+\left.\widetilde{W}\langle\nabla, v\rangle\right|_{r \rightarrow R(t)}=0
$$

которое при $r \rightarrow R(t)$ приводит к равенству

$$
\frac{\partial \widetilde{W}}{\partial R}=-\frac{\partial \widetilde{W}}{\partial r},
$$

так как $\left.v\right|_{r \rightarrow R(t)} \rightarrow d R(t) / d t:=u(t)$ и $\left.\langle\nabla, v\rangle\right|_{r \rightarrow R(t)} \rightarrow\langle\nabla, u(t)\rangle=0$ для всех $t \in \mathbb{R}$.

Возвращаясь к уравнению (25), согласно (28) мы можем записать

$$
\begin{aligned}
\left.\frac{1}{c^{2}}\left(\frac{\partial \widetilde{W}}{\partial t} v+\widetilde{W} \frac{\partial v}{\partial t}\right)\right|_{r \rightarrow R(t)} & =\left.\frac{1}{c^{2}}\left(-\left\langle\frac{\partial \widetilde{W}}{\partial r}, v\right\rangle v+\widetilde{W} \frac{\partial v}{\partial t}\right)\right|_{r \rightarrow R(t)}= \\
& =\left.\frac{1}{c^{2}}\left(\left\langle\frac{\partial \widetilde{W}}{\partial R}, u\right\rangle u+\widetilde{W} \frac{d u}{d t}\right)\right|_{r \rightarrow R(t)} \Rightarrow \\
& \Rightarrow \frac{1}{c^{2}} \frac{d}{d t}(\bar{W} u)=-\left.\frac{\partial \widetilde{W}}{\partial r}\right|_{r \rightarrow R(t)}=\frac{\partial \bar{W}}{\partial R}
\end{aligned}
$$

где мы положили $\bar{W}:=\left.\widetilde{W}(r, R(t))\right|_{r \rightarrow R_{0}}$. Таким образом, из (29) получаем, что функция $\bar{W}: \mathbb{E}^{3} \rightarrow \mathbb{R}$ удовлетворяет динамическому уравнению

$$
\frac{d}{d t}\left(-\frac{\bar{W}}{c^{2}} u\right)=-\frac{\partial \bar{W}}{\partial R}
$$

в точке $R(t) \in \mathbb{E}^{3}, t \in \mathbb{R}$, локализации точечного заряда $q$. Прежде чем перейти к нашим дальнейшим вычислениям, сделаем следующее очень важное предположение: будем интерпретировать величину $-\bar{W} / c^{2}$ как реальную динамическую массу нашего точечного заряда $q$ в точке $R(t) \in \mathbb{E}^{3}$, т.е.

$$
m:=-\frac{\bar{W}}{c^{2}} .
$$

Это, в частности, означает, что вся наблюдаемая масса частицы $m$ зависит только от вакуумного потенциала $\bar{W}$ согласно как его гравитационным, так и электромагнитным взаимодействиям с отдаленными и ближайшими, окружающими частицу другими материальными частицами. Это утверждение, очевидно, во многом совпадает с хорошо известным принципом Маха [7], [15], [17] и формализует его по отношению к реальной полевой структуре вакуума. Необходимо отметить, что эта идея была уже ранее предложена, но практически не реализована в монографии [15]. Следует подчеркнуть, что никаких предположений об эквивалентности инерциальной и гравитационной масс мы не делали. Более того, это утверждение неверно в рамках 
теории, развиваемой здесь и в работах [7], [17]. В силу (31) запишем уравнение (30) в виде

$$
\frac{d p}{d t}=-\frac{\partial \bar{W}}{\partial R}
$$

где величина $p:=m u$ имеет естественную интерпретацию импульса частицы.

Полученное уравнение (32) интересно с динамической точки зрения. Действительно, из уравнения (32) мы получаем

$$
\left\langle u, \frac{d}{d t}(m u)\right\rangle=c^{2}\left\langle\frac{\partial m}{\partial R}, u\right\rangle=c^{2} \frac{d m}{d t} .
$$

Из уравнения (33) согласно [17], [29] получаем соотношение сохранения

$$
\frac{d}{d t}\left(m \sqrt{1-\frac{u^{2}}{c^{2}}}\right)=0
$$

для всех $t \in \mathbb{R}$. Действительно, в силу (33) имеем

$$
m\left\langle u, \frac{d u}{d t}\right\rangle+\langle u, u\rangle \frac{d m}{d t}=c^{2} \frac{d m}{d t},
$$

или, эквивалентно,

$$
\frac{1}{2} m \frac{d u^{2}}{d t}-\left(c^{2}-u^{2}\right) \frac{d m}{d t}=0 .
$$

Из (36) мы получаем соотношение

$$
-\frac{1}{2} \frac{1}{1-u^{2} / c^{2}} \frac{d}{d t}\left(\frac{u^{2}}{c^{2}}\right)+\frac{1}{m} \frac{d m}{d t}=0
$$

приводящее при помощи простого интегрирования к следующему дифференциальному соотношению:

$$
\frac{d}{d t} \ln \left(\sqrt{1-\frac{u^{2}}{c^{2}}}\right)+\frac{d \ln m}{d t}=\frac{d}{d t} \ln \left(m \sqrt{1-\frac{u^{2}}{c^{2}}}\right)=0 .
$$

Соотношение (38), очевидно, эквивалентно равенству (34), т.е. величина

$$
m \sqrt{1-\frac{u^{2}}{c^{2}}}=m_{0}
$$

постоянна для всех $t \in \mathbb{R}$. Равенство (39) приводит к хорошо известному релятивистскому выражению для массы точечной частицы

$$
m=\frac{m_{0}}{\sqrt{1-u^{2} / c^{2}}} .
$$

Отсюда вытекает, что масса точечной частицы $m$ зависит на самом деле не от координаты $R(t) \in \mathbb{E}^{3}$ точечной частицы $q$, а от ее скорости $u:=d R(t) / d t$. Полевой потенциал $\bar{W}: \mathbb{E}^{3} \rightarrow \mathbb{R}$ имеет вид

$$
\bar{W}=\bar{W}_{0}+\Delta \bar{w}
$$


где $\bar{W}_{0}: \mathbb{E}^{3} \rightarrow \mathbb{R}$ - постоянной потенциал, соответствующий внешнему влиянию всех отдаленных объектов во Вселенной на точечную частицу $q$, а $\Delta \bar{w}: \mathbb{E}^{3} \rightarrow \mathbb{R}-$ потенциал, соответствующий локальному возмущению полевого потенциала точечным зарядом $q$ в его ближайшей окрестности. Тогда, очевидно,

$$
\Delta m:=m-m_{0}=-\frac{\Delta \bar{w}}{c^{2}}
$$

- компонента динамической массы, принадлежащая точечной частице $q$. Более того, так как полный импульс $p=m u$ удовлетворяет уравнению (32), получаем, что величина

$$
\bar{W}^{2}-c^{2} p^{2}=E_{0}^{2}
$$

не зависит от времени $t \in \mathbb{R}$, т.е. $d E_{0} / d t=0$, где $E_{0}:=m_{0} c^{2}-$ внутренняя энергия точечной частицы $q[5],[8],[20]$, [30]. Результат (43) показывает важное свойство понятия энергии: точечная частица $q$ в действительности наделена только динамической энергией $\Delta E:=\Delta m c^{2}$. Часть внутренней энергии частицы $E_{0}=m_{0} c^{2}$ не имеет никакого отношения к реальной энергии частицы, так как ее происхождение полностью определяется отдаленными объектами Вселенной и она не может быть использована для описания каких-либо физических процессов, в противоположность хорошо известным утверждениям теории Эйнштейна о гигантской внутренней энергии, запасенной внутри массы частицы. В то же время утверждение теории Эйнштейна об эквивалентности массы и внутренней энергии частицы также не имеет смысла, так как главная часть полевой потенциальной функции $\bar{W}: \mathbb{E}^{3} \rightarrow \mathbb{R}$ в месте локализации точечной частицы $q$ постоянна и является результатом действия отдаленных объектов во Вселенной, что, очевидно, не может быть использовано на практике. Тем не менее выше мы получили как побочный результат хорошо известный релятивистский эффект роста массы частицы в зависимости от скорости частицы (см. (40)). Как уже было отмечено в работах [7], [17], этот рост массы носит в действительности полностью динамический характер и не является следствием преобразований Лоренца, как это утверждалось в СТО Эйнштейна. Более того, мы можем утверждать, что все так называемые релятивистские эффекты не имеют ничего общего ни с приведенными выше преобразованиями Лоренца, ни с таким искусственным эффектом как "сокращение" длины и “замедление" времени. Нет также разумной причины отождествлять массу частицы и ее энергию и наоборот. Касаясь интересного физического эффекта, называемого аннигиляцией частиц, необходимо подчеркнуть, что он не имеет никакого отношения к превращению масс частиц в энергию. Теоретико-полевое объяснение этого эффекта состоит в создании такого специального связанного состояния, когда нет взаимодействия с окружающими объектами. В результате наблюдаемая инертная или динамическая масса этого связанного состояния также нулевая, что и показывает эксперимент. И наоборот, если достаточно интенсивный гамма-квант встречает такое связанное состояние двух частиц, он может разбить его опять на две отдельные частицы. То, что это происходит, показывает эксперимент. Здесь мы можем также вспомнить аналогию из современной квантовой физики бесконечных систем частиц, описываемых 
при помощи вторичного квантования [29], [31]-[33], предложенного Фоком в 1932 г. В этом случае также реализуются эффекты рождения и уничтожения частиц, которые имеют место вследствие межчастичных сил взаимодействия. Более того, как мы знаем из современных теорий сверхтекучести и сверхпроводимости, таким же образом можно описать специальные связанные состояния частиц - так называемые куперовские пары, взаимодействие которых между собой полностью исчезает и чья объединенная масса существенно отличается от соответствующих компонент и равняется так называемой эффективной составной массе, зависящей от интенсивности потенциального поля внутри сверхтекучей или сверхпроводящей среды.

Перейдем теперь к обсуждению полученного выше соотношения (43), где сохраняющаяся величина $E_{0}$ интерпретируется как полная энергия частицы, движущейся со скоростью $u:=d R(t) / d t$ в вакууме, наделенном полевым потенциалом $\bar{W}$ в окрестности частицы, локализованной в точке $R(t) \in \mathbb{E}^{3}$. Из соотношения (43) видно, что полная энергия частицы $E_{0}$ зависит как от полевого потенциала $\bar{W}$, так и от скорости $u$, так как импульс частицы $p=m_{0} u / \sqrt{1-u^{2} / c^{2}}$ релятивистски зависит от скорости $u$.

Как было отмечено в монографии [15], в СТО Эйнштейна постулируется, что полная энергия $E_{0}$ движущейся частицы в потенциальном поле $U$ равняется $E_{0}=$ $m_{0} c^{2} / \sqrt{1-u^{2} / c^{2}}+U$. Бриллюэн ([15], с. 31) пишет: “Это означает, что всякая возможность существования у тела массы, связанной с внешней потенциальной энергией, полностью исключается. ... Таким образом, получается странная ситуация: внутренней энергии отвечает масса, а внешней - нет".

В противоположность этому выводу, соотношение (43), полученное выше, учитывает как кинетическую энергию движения частицы со скоростью $u$, так и полевую потенциальную энергию $\bar{W}$ в окрестности частицы $q$, локализованной в точке $R(t) \in \mathbb{E}^{3}$.

Более того, Бриллюэн ([15], с. 77) пишет: “Эйнштейн во что бы то ни стало стремится свести гравитацию к геометрии путем замены ньютоновского гравитационного потенциала тензорным потенциалом второго порядка, осуществляющим совместное описание гравитации и геометрии: это достигается ценой появления пропасти между гравитацией и электромагнетизмом. Цитируемая статья (Эйнштейна) гениальная математическая работа, однако вопрос о ее применении к физической реальности остается открытым". Позже Фок в своем знаменитом труде [20] попытался спасти ситуацию, которая возникла с гравитацией и электромагнетизмом, но его подход был основан на идеях геометризации Эйнштейна и реального успеха не было достигнуто. Оценивая состояние теории гравитации и электромагнетизма, Бриллюэн ([15], с. 84) утверждает: “В общем, необходимость рассмотрения искривленного пространственно-временного мира еще не доказана; физическое значение общей теории относительности пока очень неясно".

Проанализировав с этой точки зрения результаты, сформулированные выше, мы видим, что все утверждения СТО Эйнштейна были получены для евклидова пространства-времени и без применения четырехмерной геометрии пространства-времени типа инвариантности четырехмерного метрического интервала по отношению к пре- 
образованиям Лоренца. Ниже мы покажем, что в рамках подхода, развитого выше и в работах [7], [17], можно получить очень важный результат СТО и теории электромагнетизма Максвелла, а именно результат, связанный с природой силы Лоренца, действующей на движущуюся заряженную частицу в пространстве. Как хорошо известно [15], [17], [18], [20], желание получить выражение для силы Лоренца, согласованное с постулированными принципами релятивизма, оказалось решающим при попытках Эйнштейна построить СТО и позже общую теорию относительности. Как очень интересный аспект полевой теории вакуума, предложенной выше и в работах [7], [17], необходимо отметить очень близкую связь электромагнитного и гравитационного полей. А именно, вакуумный полевой потенциал в общем случае состоит из двух компонент: $W:=W_{\mathrm{g}}+W_{\mathrm{em}}$, где $W_{\mathrm{g}}$ описывает гравитационное взаимодействие между материальными частицами, а $W_{\text {em }}$ - электромагнитное взаимодействие между их зарядами. При этом мы считаем, что эти две вакуумные физические характеристики являются различными и независимыми. Соответствующая полная масса частицы дается тогда выражением (31) в виде

$$
m:=-\frac{\bar{W}}{c^{2}}=-\frac{\bar{W}_{\mathrm{g}}+\bar{W}_{\mathrm{em}}}{c^{2}},
$$

включающим как гравитационные, так и электромагнитные взаимодействия между частицами. Тогда, следуя выводу уравнений электромагнитного поля Максвелла, основанному на электромагнитном вакуумном полевом потенциале $W:=W_{\mathrm{em}}$, мы можем вывести аналогичным образом соответствующие гравимагнитные уравнения типа уравнений Максвелла для гравитационного потенциала $W_{\mathrm{g}}$ :

$$
\begin{aligned}
& \frac{1}{c^{2}} \frac{\partial^{2} W_{\mathrm{g}}}{\partial t^{2}}-\nabla^{2} W_{\mathrm{g}}=\rho_{\mathrm{g}}, \quad \frac{1}{c^{2}} \frac{\partial^{2} A_{\mathrm{g}}}{\partial t^{2}}-\nabla^{2} A_{\mathrm{g}}=J_{\mathrm{g}}, \\
& \nabla \times B_{\mathrm{g}}-\frac{1}{c} \frac{\partial E_{\mathrm{g}}}{\partial t}=\frac{1}{c} J_{\mathrm{g}}, \quad \frac{1}{c} \frac{\partial B_{\mathrm{g}}}{\partial t}-\nabla \times E_{\mathrm{g}}=0, \\
& \left\langle\nabla, E_{\mathrm{g}}\right\rangle=\rho_{\mathrm{g}}, \quad\left\langle\nabla, B_{\mathrm{g}}\right\rangle=0,
\end{aligned}
$$

где

$$
E_{\mathrm{g}}:=-\nabla W_{\mathrm{g}}-\frac{1}{c} \frac{\partial A_{\mathrm{g}}}{\partial t}, \quad B_{\mathrm{g}}:=\nabla \times A_{\mathrm{g}},
$$

$\rho_{\mathrm{g}}$ и $J_{\mathrm{g}}-$ плотность гравитационного заряда массы частицы и плотность массового гравитационного тока соответственно. Вид уравнений (45) связан с хорошо известным подобием между выражениями для электрических кулоновских и гравитационных ньютоновских сил и раньше обсуждался в работах [3], [15], [17], [34].

Как было показано выше, в консервативном случае имеют место следующие представления типа (18):

$$
J_{\mathrm{g}}=\rho_{\mathrm{g}} v, \quad A_{\mathrm{g}}=\frac{v}{c} W_{\mathrm{g}} .
$$

Как следствие, мы можем утверждать, что гравитационные волны существуют, распространяясь в вакууме с той же самой скоростью света $c$, как и электромагнитные волны, и описываются при помощи гравимагнитных уравнений типа уравнений Максвелла (45). Отметим, что подобные выводы были сделаны много лет назад в классических трудах знаменитых физиков XIX века Максвелла [35] и Хевисайда [36], а также в работах других физиков [17], [19], [34], [37], [38]. 


\section{4. СИЛА ЛОРЕНЦА И ПРИНЦИПЫ РЕЛЯТИВИСТСКОЙ ТЕОРИИ: НОВЫЙ ПОДХОД}

Хорошо известно [5], [8], что СТО Эйнштейна применима только для физических процессов, связанных между собой посредством инерциальных систем отсчета, движущихся с постоянными скоростями. В этом случае можно воспользоваться преобразованиями Лоренца и сосчитать компоненты соответствующих 4-векторов и результирующий рост массы частиц согласно формуле (33). Но возникает нетривиальная проблема, когда мы пытаемся анализировать эти величины по отношению к неинерциальным системам отсчета, движущимся с ненулевым ускорением. Ниже мы возобновим анализ этой проблемы на основе теоретико-полевого метода описания вакуума, предложенного выше, и покажем, что СТО появляется как его частный случай, или даже как следствие, и оказывается свободной от проблем, связанных с искусственно выделенными инерциальными системами отсчета.

Действительно, наша теоретико-полевая структура вакуума описывается динамическим уравнением (25), которое мы будем исследовать в окрестности двух взаимодействующих точечных частиц $q_{f}$ в точке $R_{f}(t) \in \mathbb{E}^{3}$ и $q$ в точке $R(t):=$ $R_{0}+\int_{0}^{t} u(t) d t \in \mathbb{R} 3$, зависящих от параметра $t \in \mathbb{R}$ и начальной точки $R_{0} \in \mathbb{E}^{3}$ при $t=0$. Как и в разделе 2 , мы полагаем, что полевая потенциальная функция вакуума $W: \mathbb{E}^{3} \times \mathbb{R} \rightarrow \mathbb{R}$ может быть представлена как $W=\widetilde{W}\left(r ; R_{f}(t), R(t)\right)$ для некоторой функции $\widetilde{W}: \mathbb{E}^{3} \times \mathbb{E}^{3} \times \mathbb{E}^{3} \rightarrow \mathbb{R}$ и всех $t \in \mathbb{R}$.

Тогда согласно уравнению непрерывности (17) мы получаем

$$
\left\langle\frac{\partial \widetilde{W}}{\partial R_{f}}, u_{f}\right\rangle+\left\langle\frac{\partial \widetilde{W}}{\partial R}, u\right\rangle+\left\langle\frac{\partial \widetilde{W}}{\partial r}, v\right\rangle+\widetilde{W}\langle\nabla, v\rangle=0
$$

Теперь нас будет интересовать потенциальная полевая функция $\widetilde{W}: \mathbb{E}^{3} \times \mathbb{E}^{3} \times \mathbb{E}^{3} \rightarrow \mathbb{R}$ в окрестности вектора относительного расстояния $\widetilde{R}(t):=R(t)-R_{f}(t) \in \mathbb{E}^{3}$ при условии, что взаимодействие между частицами $q_{f}$ и $q$ зависит от относительного межчастичного расстояния $\widetilde{R}(t) \in \mathbb{E}^{3}$. Из (48) при $r \rightarrow \widetilde{R}(t)$ мы легко выводим следующие соотношения:

$$
\frac{\partial \widetilde{W}}{\partial R_{f}}+\left.\frac{\partial \widetilde{W}}{\partial R}\right|_{r \rightarrow \widetilde{R}(t)}=0, \quad \frac{\partial \widetilde{W}}{\partial R}+\left.\frac{\partial \widetilde{W}}{\partial r}\right|_{r \rightarrow \widetilde{R}(t)}=0 .
$$

Объединяя соотношения (49) с динамическими полевыми уравнениями (25), мы получаем

$$
\begin{aligned}
\left.\frac{1}{c^{2}} \frac{\partial}{\partial t}(\widetilde{W} v)\right|_{r \rightarrow \widetilde{R}(t)} & =\left.\frac{1}{c^{2}}\left(\left\langle\frac{\partial \widetilde{W}}{\partial R_{f}}, u_{f}\right\rangle v+\left\langle\frac{\partial \widetilde{W}}{\partial R}, u\right\rangle v+\widetilde{W} \frac{\partial v}{\partial t}\right)\right|_{r \rightarrow \widetilde{R}(t)}= \\
& =\left.\frac{1}{c^{2}}\left(\left\langle\frac{\partial \widetilde{W}}{\partial \widetilde{R}}, u-u_{f}\right\rangle\left(u-u_{f}\right)\right)\right|_{r \rightarrow \widetilde{R}(t)}= \\
& =-\left.\frac{\partial \widetilde{W}}{\partial r}\right|_{r \rightarrow \widetilde{R}(t)}=\left.\frac{\partial \widetilde{W}}{\partial R}\right|_{r \rightarrow \widetilde{R}(t)},
\end{aligned}
$$


откуда имеем новое динамическое уравнение

$$
\frac{d}{d t}\left(-\frac{\bar{W}}{c^{2}}\left(u-u_{f}\right)\right)=-\frac{\partial \bar{W}}{\partial R}
$$

на результирующую функцию $\bar{W}:=\left.\widetilde{W}\right|_{r \rightarrow \widetilde{R}(t)}$.

Уравнение (50) обладает очень важным свойством зависимости только от относительных величин, не зависящих от системы отсчета. Более того, нам вообе не потребовалось использовать каких-либо преобразований координат, отличных от обычных галилеевских. Отметим, что динамическое уравнение (50) также было выведено в монографии [17], но с использованием не вполне корректных соотношений и математических преобразований. Из работ [17] и [7] вытекает, что уравнение (50) справедливо для всех систем отсчета, как инерциальных, так и движущихся с ускорением, оказывается фундаментальным и приводит к новым неожиданным результатам в современной электродинамике и теории гравитации. Ниже мы перейдем к анализу одного очень важного аспекта релятивистской физики, касающегося хорошо известного выражения для силы Лоренца, описывающей меру воздействия внешнего электромагнитного поля на заряженную точечную частицу $q$ в пространственной точке $R(t) \in \mathbb{E}^{3}$ для любого момента времени $t \in \mathbb{R}$.

Согласно развитой полевой теории вакуума результирующая потенциальная полевая функция $\bar{W}: \mathbb{E}^{3} \rightarrow \mathbb{R}$ может быть представлена в окрестности заряженной точечной частицы $q$ в виде

$$
\bar{W}=\bar{W}_{0}+q \varphi
$$

где $\varphi: \mathbb{E}^{3} \rightarrow \mathbb{R}-$ соответствующий локальный электромагнитный полевой потенциал, $\bar{W}_{0}: \mathbb{E}^{3} \rightarrow \mathbb{R}$ - постоянный вакуумный полевой потенциал, соответствующий взаимодействию частицы с внешними отдаленными объектами Вселенной. Подставив (51) в основное динамическое уравнение (50), получаем

$$
\begin{aligned}
\frac{d}{d t}\left(-\frac{\bar{W}}{c^{2}} u\right) & =\frac{d}{d t}\left(-\frac{\bar{W}}{c^{2}} u_{f}\right)-\nabla \bar{W}=-\nabla \bar{W}+\frac{\partial}{\partial t}\left(-\frac{\bar{W}}{c^{2}} u_{f}\right)+\langle u, \nabla\rangle\left(-\frac{\bar{W}}{c^{2}} u_{f}\right)= \\
& =-\nabla \bar{W}+\frac{1}{c} \frac{\partial}{\partial t}\left(-\frac{\bar{W}}{c} u_{f}\right)-u \times\left(u_{f} \times \nabla \frac{\bar{W}}{c^{2}}\right)-\left\langle u, u_{f}\right\rangle \nabla \bar{W}= \\
& =-\nabla \bar{W}\left(1+\frac{\left\langle u, u_{f}\right\rangle}{c^{2}}\right)+\frac{1}{c} \frac{\partial}{\partial t}\left(-\frac{\bar{W}}{c} u_{f}\right)-\frac{1}{c^{2}} u \times\left(u_{f} \times \nabla \bar{W}\right)= \\
& =-q \nabla \varphi\left(1+\frac{\left\langle u, u_{f}\right\rangle}{c^{2}}\right)-\frac{q}{c} \frac{\partial}{\partial t}\left(\frac{\varphi}{c} u_{f}\right)+\frac{q}{c} u \times\left(\nabla \times \frac{\varphi u_{f}}{c}\right)= \\
& =-q \nabla \varphi\left(1+\frac{\left\langle u, u_{f}\right\rangle}{c^{2}}\right)-\frac{q}{c} \frac{\partial A}{\partial t}+\frac{q}{c} u \times(\nabla \times A)
\end{aligned}
$$

где $u:=d R(t) / d t, u_{f}:=d R_{f}(t) / d t, \nabla:=\partial / \partial R=-\partial / \partial R_{f}$, а $A:=\varphi u_{f} / c-$ соответствующий магнитный потенциал. Поскольку мы уже показали, что сила Лоренца

$$
F:=\frac{d}{d t}\left(-\frac{\bar{W}}{c^{2}} u\right)=\frac{d}{d t}\left(\frac{m_{0} u}{\sqrt{1-u^{2} / c^{2}}}\right)
$$


дается выражением (52), теперь оно может быть переписано в виде (см. также [17])

$$
\begin{aligned}
F & =\frac{d}{d t}\left(\frac{m_{0} u}{\sqrt{1-u^{2} / c^{2}}}\right)=q E+\frac{q}{c} u \times B-\frac{q}{c^{2}} \nabla \varphi\left\langle u, u_{f}\right\rangle= \\
& =q E+\frac{q}{c} u \times B-\frac{q}{c} \nabla\langle u, A\rangle,
\end{aligned}
$$

где $E:=-\nabla \varphi-(1 / c)(\partial A / \partial t), B:=\nabla \times A-$ электрическое и магнитное векторные поля соответственно.

Результирующее выражение (53) почти полностью эквивалентно хорошо известному [11] выражению для классической силы Лоренца $F$ с точностью до дополнительного инерциального члена

$$
F_{\mathrm{c}}:=-\frac{q}{c} \nabla\langle u, A\rangle,
$$

который отсутствует в СТО Эйнштейна. Именно в силу отсутствия члена (54) классическое выражение для релятивистской силы Лоренца в 4-векторной форме оказалось неинвариантным по отношению к любым преобразованиям системы отсчета, кроме инерциальных. Как было отмечено в работах [15], [17], только благодаря этому решающему факту Эйнштейн ввел преобразования Лоренца и ассоциированные с ними видимые эффекты сокращения длины и замедления времени. Более того, они привели к таким достаточно странным и неадекватным понятиям, как неевклидово пространство-время [15], [39]-[42], черные дыры [6], [39], [43], [44] и некоторые другие сингулярные и нефизические объекты. В силу (53) мы можем легко получить соответствующий закон сохранения энергии для движущейся заряженной точечной частицы $q$ и окружающего магнитного поля:

$$
\frac{d E_{0}}{d t}=0
$$

где

$$
E_{0}:=\left(\bar{W}^{2}-c^{2} P^{2}\right)^{1 / 2}+\frac{q}{c}\langle A, P\rangle\left(\bar{W}^{2}-c^{2} P^{2}\right)^{-1 / 2},
$$

$P:=p-q A / c$ - "сдвинутый" импульс заряженной точечной частицы $q$ во внешнем магнитном поле, $B=\nabla \times A$, параметр $R_{f}:=\left.R_{f}(t)\right|_{t=t_{f}} \in \mathbb{E}^{3}$, а параметр эволюции $t_{f}=t \in \mathbb{R}$ отвечает за изменение только магнитной потенциальной энергии $E_{f}$. Полученные выражения $(55)$ и (56) учитывают, очевидно, естественный баланс энергий, принадлежащих как движущейся заряженной точечной частице $q$, так и окружающему магнитному полю.

\section{5. КВАНТОВАЯ МЕХАНИКА: ВОЗВРАТ К ОСНОВАНИЯМ}

Рассмотрим соотношение (43) в виде

$$
E_{0}^{2}=\bar{W}-p^{2} c^{2}, \quad \frac{d E_{0}}{d t}=0 .
$$

Сделаем следующие подстановки канонического квантования:

$$
p \rightarrow \hat{p}:=\frac{\hbar}{i} \nabla, \quad E_{0} \rightarrow \widehat{E}_{0}:=-\frac{\hbar}{i} \frac{\partial}{\partial t}, \quad \bar{W} \rightarrow \bar{W} \circ,
$$


где $\hat{p}=(\hbar / i) \nabla$ - стандартный генератор пространственных трансляций в эвклидовом пространстве $\mathbb{E}^{3}, \widehat{E}_{0}=-(\hbar / i)(\partial / \partial t)$ - стандартный оператор трансляций вдоль действительной оси времени $\mathbb{R}, \bar{W}$ о - обычный скалярный оператор умножения на функцию $\bar{W}: \mathbb{E}^{3} \rightarrow \mathbb{R}$, действующие в гильбертовом пространстве $\mathcal{H}:=L_{2}\left(\mathbb{E}^{3} ; \mathbb{C}\right)$ со скалярным произведением $(\cdot, \cdot)$. Как результат этой замены согласно методам квантования, подобным используемым в работах [9]-[11], [30], мы можем записать наблюдаемый квадрат энергии $E_{0}^{2}$ как среднее значение

$$
E_{0}^{2}:=\left(\widehat{E}_{0} \psi, \widehat{E}_{0} \psi\right)=\left(\psi,\left(\bar{W}^{2}+\hbar^{2} c^{2} \nabla^{2}\right) \psi\right),
$$

реализуемое на одночастичном нормированном квантово-механическом векторе состояния $\psi \in \mathcal{H}$. Применяя представление (59) и предполагая, что постоянная Планка $\hbar \rightarrow 0$, можно попытаться факторизовать линейный дифференциальный оператор $\bar{W}^{2}+\hbar^{2} c^{2} \nabla^{2}$, определенный на соответствующем плотном линейном подмножестве $D\left(\widehat{E}_{0}\right) \subset \mathcal{H}$, в следующей неотрицательной канонической форме:

$$
\bar{W}^{2}+\hbar^{2} c^{2} \nabla^{2}=\widehat{P}^{+} \widehat{P}
$$

где индекс “+" означает стандартную операцию сопряжения в гильбертовом пространстве $\mathcal{H}$. Из (60) находим

$$
\widehat{P}:=\widehat{S}\left(1+\hbar^{2} c^{2} \bar{W}^{-1} \circ \nabla^{2} \circ \bar{W}^{-1}\right)^{1 / 2} \bar{W} \circ
$$

с произвольным унитарным оператором $\widehat{S}: \mathcal{H} \rightarrow \mathcal{H}$, удовлетворяющим условию $\widehat{S}^{+} \widehat{S}=1$. Из (59) и (60) получаем

$$
\left(\widehat{E}_{0} \psi, \widehat{E}_{0} \psi\right)=\left(\psi, \widehat{P}^{+} \widehat{P} \psi\right)=(\widehat{P} \psi, \widehat{P} \psi) .
$$

Тем самым в силу выражения (62) мы можем вывести следующее линейное эволюционное уравнение типа уравнения Шредингера:

$$
\widehat{E}_{0} \psi:=i \hbar \frac{\partial \psi}{\partial t}=\widehat{U} \widehat{P} \psi
$$

для всех $t \in \mathbb{R}$, где $\widehat{U}: \mathcal{H} \rightarrow \mathcal{H}$ - некоторый унитарный оператор, который будет определен позже. Теперь мы вычислим операторное выражение (61) с символической операторной точностью $O\left(\hbar^{4}\right)$ :

$$
\widehat{P}=\widehat{S}\left(1+\hbar^{2} c^{2} \bar{W}^{-1} \circ \nabla^{2} \circ \bar{W}^{-1}\right)^{1 / 2} \bar{W}=\widehat{S}\left(1+\frac{\hbar^{2} c^{2}}{2 \bar{W}} \nabla^{2} \circ \bar{W}^{-1}\right) \bar{W}+O\left(\hbar^{4}\right) .
$$

Подставив результат (64) в (63), получаем (с операторной точностью $\left.O\left(\hbar^{4}\right)\right)$ следующее эволюционное уравнение:

$$
i \hbar \frac{\partial \psi}{\partial t}=\widehat{U} \widehat{S}\left(-\frac{\hbar^{2}}{2\left(-\bar{W} / c^{2}\right)} \nabla^{2}+\bar{W}\right) \psi
$$

Учитывая, что оператор энергии $\widehat{E}_{0}=-(\hbar / i)(\partial / \partial t)$ является формально самосопряженным, с физической точки зрения мы требуем выбрать в (65) $\widehat{U}:=\widehat{S}^{-1}$, так как 
оператор $\widehat{H}:=-\left(\hbar^{2} /\left(2\left(-\bar{W} / c^{2}\right)\right)\right) \nabla^{2}+\bar{W}$ является тоже формально самосопряженным.

В силу (31) и (40) выражение $-\bar{W} / c^{2}:=m(u)=m_{0} / \sqrt{1-u^{2} / c^{2}}$ - динамическая масса частицы, квантовое движение которой мы изучаем. Уравнение (65) в конечном итоге может быть переписано в следующей классической самосопряженной форме уравнения Шредингера:

$$
i \hbar \frac{\partial \psi}{\partial t}=\widehat{H} \psi:=-\frac{\hbar^{2}}{2 m(u)} \nabla^{2} \psi+\bar{W} \psi
$$

где $\widehat{H}: \mathcal{H} \rightarrow \mathcal{H}$ - соответствующий самосопряженный оператор Гамильтона для релятивистской точечной частицы во внешнем потенциальном поле. Легко заметить, что уравнение (66) в классическом нерелятивистком случае при $c \rightarrow \infty$ приводится к хорошо известному классическому уравнению Шредингера

$$
i \hbar \frac{\partial \psi}{\partial t}=\widehat{H}_{0} \psi:=-\frac{\hbar^{2}}{2 m_{0}} \nabla^{2} \psi+\bar{W} \psi
$$

полученному в начале XX века из разных соображений Шредингером, Гейзенбергом и Дираком.

Теперь мы перейдем к подобной процедуре квантования выражения (56) для случая, когда магнитный потенциал не является нулевым. Используя стандартную схему квантования (58), мы получаем, что значение среднего квадрата энергии $E_{0}^{2}$ на соответствующем нормированном квантовом векторе состояния $\psi \in \mathcal{H}$ равно

$$
\begin{aligned}
E_{0}^{2}:=\left(\widehat{E}_{0} \psi, \widehat{E}_{0} \psi\right) & =\left(\psi,\left[\widehat{W}^{2}-c^{2}\left(\frac{\hbar}{i} \nabla-\frac{q}{c} \hat{A}\right)^{2}\right]+\right. \\
& \left.\left.+\left(\frac{q}{c}\right)^{2} \hat{A}^{2}+\left(\frac{q}{c}\right)^{2}\left\langle\hat{A}, \frac{\hbar}{i} \nabla\right\rangle\left[\widehat{W}^{2}-c^{2}\left(\frac{\hbar}{i} \nabla\right)^{2}\right]^{-1}\left\langle\frac{\hbar}{i} \nabla, \hat{A}\right\rangle\right] \psi\right),
\end{aligned}
$$

где $\widehat{W}:=\bar{W}$ о и $\hat{A}:=A \circ-$ скалярные операторы умножения. Операторные выражения в квадратных скобках (68) могут быть представлены в следующей существенно неотрицательной форме:

$$
\widehat{W}^{2}-c^{2}\left(\frac{\hbar}{i} \nabla-\frac{q}{c} \hat{A}\right)^{2}+\left(\frac{q}{c}\right)^{2}\left\langle\hat{A}, \frac{\hbar}{i} \nabla\right\rangle\left[\widehat{W}^{2}-c^{2}\left(\frac{\hbar}{i} \nabla\right)^{2}\right]^{-1}\left\langle\frac{\hbar}{i} \nabla, \hat{A}\right\rangle:=\widehat{P}^{+} \widehat{P}
$$

где

$$
\begin{aligned}
\widehat{P}:= & 1-\widehat{W}^{-1}\left[c^{2}\left(\frac{\hbar}{i} \nabla-\frac{q}{c} \hat{A}\right)^{2}-\left(\frac{q}{c}\right)^{2} \hat{A}^{2}-\right. \\
& \left.-\left(\frac{q}{c}\right)^{2}\left\langle\hat{A}, \frac{\hbar}{i} \nabla\right\rangle\left[\widehat{W}^{2}-c^{2}\left(\frac{\hbar}{i} \nabla\right)^{2}\right]^{-1}\left\langle\frac{\hbar}{i} \nabla, \hat{A}\right\rangle \widehat{W}^{-1}\right)^{1 / 2} \widehat{W} .
\end{aligned}
$$

Тогда из уравнения (68) и выражения (69) мы можем записать магнитное эволюционное уравнение типа Шредингера:

$$
i \hbar \frac{\partial \psi}{\partial t}:=\widehat{P} \psi
$$


которое приводит при одновременном выполнении условий $\hbar \rightarrow 0, c \rightarrow \infty$ и $c \hbar=$ const к следующему результату:

$$
i \hbar \frac{\partial \psi}{\partial t}:=\widehat{H} \psi=\left[\frac{1}{2 m(u)}\left(\frac{\hbar}{i} \nabla+\frac{q}{c} \hat{A}\right)^{2}+\bar{W}_{0}+q \varphi\right] \psi
$$

с точностью $O\left(\hbar^{4}\right)$. Уравнение (72) редуцируется к хорошо известному [30] классическому магнитному эволюционному уравнению типа Шредингера с точностью $O\left(\hbar^{4}\right)$ :

$$
i \hbar \frac{\partial \psi}{\partial t}:=\widehat{H}_{0} \psi=\left[\frac{1}{2 m_{0}}\left(\frac{\hbar}{i} \nabla-\frac{q}{c} \hat{A}\right)^{2}+W_{0}+q \varphi\right] \psi,
$$

где $m_{0}=-\bar{W}_{0} / c^{2}$, так как имеет место соотношение для потенциальной энергии $\left|q \varphi / \bar{W}_{0}\right| \ll 1$.

Полученные выше результаты показывают, что наш квазиклассический подход к описанию вакуумной полевой структуры, когда постоянная Планка $\hbar \rightarrow 0$ и скорость света $c \rightarrow \infty$, естественно приводит к ассоциированной квантовой динамике Шредингера, оставаясь при этом одновременно глубоко физически мотивированным. Важно также отметить, что во всех выводах мы не использовали лоренцевой инвариантности, несмотря на тот факт, что динамическая масса $m=$ $m_{0} / \sqrt{1-u^{2} / c^{2}}$ выражается с помощью преобразования Лоренца. Это, очевидно, побочный результат уравнения (2) второго теоретико-полевого принципа, который является лоренц-инвариантным.

В заключение отметим, что полностью релятивистский квантово-полевой теоретический анализ нашей динамической полевой модели вакуума с помощью хорошо известного метода факторизации Дирака [30] вполне возможно прояснит сложную и загадочную природу квантового мира элементарных частиц. Учитывая полученные результаты, можно утверждать, что теоретико-полевой вакуумный подход [3], [7], [13], [15], [17], [18], [25] к фундаментальным физическим явлениям оказывается действительно эффективным методом для исследователей, которые пытаются изучить свойства окружающей Вселенной. Поскольку микроскопический квантовый уровень описания структуры вакуумной полевой среды, безусловно, весьма важен, мы надеемся предпринять исследования как в направлении понимания основ квантовых процессов в пределах подходов, развитых в работах [7], [15], [17] и в настоящей работе, так и в выводе новых физических соотношений, основанных на канонических формализмах Лагранжа и Гамильтона.

Благодарности. Н. Н. Боголюбов (мл.) и А. К. Прикарпатский сердечно благодарны Международному центру теоретической физики им. Абдуса Салама (Триест, Италия) за гостеприимство во время их исследовательской работы в 2007-2008 гг. А.К. Прикарпатский также очень признателен Б. А. Дубровину (Триест, ШВИ), П. И. Голоду (Киев, УКМА), И. М. Стахире (Львов, ЛНУ), Б. М. Барбашову (Дубна, ОИЯИ), З. Конколю (Краков, АГПМ), Я. Славяновскому (Варшава, ИФПТ), З. Перадзинскому (Варшава, ВУ) и М. Блашаку (Познань, ПУ) за полезные обсуждения, ценные комментарии и замечания. Авторы выражают особенную благодарность 
А. А. Логунову (Москва, ИФВЭ) за проявленный интерес к работе, О. Н. Репченко (Москва) за обсуждения некоторых аспектов полевой теории вакуума, а также Дилыс Грилли (Триест, издательский отдел, МЦТФ) за профессиональную помощь при подготовке рукописи к печати.

\section{Список литературы}

[1] R. P. Feynman et al., "The Feynman Lectures on Gravitation", (Based on notes by F. B. Morinigo and W. G. Wagner, edited by B. Hatfield), Addison-Wesley, Reading, MA, 1995.

[2] P. G. de Gennes, Superconductivity of Metals and Alloys, Benjamin, New York, 1966.

[3] J. Carstoiu, C. R. Acad. Sci. Paris, 268 (1969), 201-204.

[4] М.А. Марков, "Принцип Маха и физический вакуум в общей теории относительности", Проблемы теоретической физики, Сборник, посвящ. 60-летию Н. Н. Боголюбова, ред. Д. И. Блохинцев, Наука, М., 1969, 26-27.

[5] E. F. Taylor, J. A. Wheeler, Spacetime Physics, Freeman, New York, 1992.

[6] B. M. Barbashov, D. Blaschke, G. V. Efimov et al., Selected Problems of Modern Physics, Proc. of the XII-th Internat. Conf., Sect. 1, Dubna, 2003.

[7] A. K. Prykarpatsky, N. N. Bogolubov Jr., The field structure of vacuum, Maxwell equations and relativity theory aspects. Part 1, arXiv: 0807.3691.

[8] Р. Фейнман, Р. Лейтон, М. Сэндс, Фейнмановские лекиии по физике. Т. 6. Электродинамика, Мир, М., 1977.

[9] Н. Н. Боголюбов, Д. В. Ширков, Введение в теорию квантованных полей, Наука, М., 1973.

[10] Дж. Д. Бьеркен, С. Д. Дрелл, Релятивистская квантовая теория. Т. II. Релятивистские квантовые поля, Наука, М., 1978.

[11] А. И. Ахиезер, В. Б. Берестецкий, Квантовая электродинамика, Наука, М., 1981.

[12] J. Schwinger, Quantum Electrodynamics, Dover, New York, 1958.

[13] I. Bialynicki-Birula, Phys. Rev., 155:5 (1967), 1414-1414; 166:5 (1968), 1505-1506.

[14] А. Зоммерфельд, Механика, РХД, Ижевск, 2001.

[15] Л. Бриллюэн, Новый взгляд на теорию относительности, Мир, М., 1972.

[16] Л. Д. Фаддеев, УФН, 136:3 (1982), 435-457.

[17] О.Н. Репченко, Полевая физика или как устроен Мир?, Галерия, М., 2005.

[18] C. H. Brans, R. H. Dicke, Phys. Rev., 124:3 (1961), 925-935.

[19] А.А. Логунов, Лекиии по теории относительности и гравитации, Наука, М., 1987.

[20] В. А. Фок, Теория пространства, времени и тяготения, Наука, М., 1961.

[21] В. Паули, Теория относительности, Наука, М., 1983.

[22] R. Weinstock, Amer. J. Phys., 33:8 (1965), 640-645.

[23] A. R. Lee, T. M. Kalotas, Amer. J. Phys., 43:5 (1975), 434-437.

[24] J. M. Lévy-Leblond, Amer. J. Phys., 44:3 (1976), 271-277.

[25] N.D. Mermin, Amer. J. Phys., 52:2 (1984), 119-124.

[26] A. Sen, Amer. J. Phys., 62:2 (1994), 157-162.

[27] P. W. Bridgman, Reflections of a Physicist, Philosophical Library, New York, 1950.

[28] A. Chorin, J. Marsden, A Mathematical Introduction to Fluid Mechanics, Texts Appl. Math., 4, Springer, New York, 1993.

[29] A. K. Prykarpatsky, N. N. Bogolubov (Jr.), J. Golenia, U. Taneri, Internat. J. Theoret. Phys., 47:11 (2008), 2882-2897; publications.ictp.it.

[30] П.А. М. Дирак, Принципы квантовой механики, Физматгиз, М., 1960.

[31] V.A. Fock, Z. Phys., 75:9-10 (1932), 622-647.

[32] Ф.А. Березин, Метод вторичного квантования, Наука, М., 1965. 
[33] A. K. Prykarpatsky, U. Taneri, N. N. Bogolubov Jr., Quantum Field Theory with Application to Quantum Nonlinear Optics, World Scientific, Singapore, 2002.

[34] А. З. Петров, Докл. АН УССР, 190 (1970), 305.

[35] Дж. К. Максвелл, Трактат об электричестве и магнетизме, Т. 1, 2, Наука, М., 1989.

[36] O. Heaviside, Electromagnetic Theory, V. I, The Electrician Printing, London, 1894.

[37] L. Brillouin, R. Lucas, J. Phys. Radium, 27 (1966), 229-232.

[38] R. Burghardt, Acta Phys. Austr., 32 (1970), 272-281.

[39] G. t'Hooft, Introduction to General Relativity, Rinton Press, Princeton, NJ, 2001;

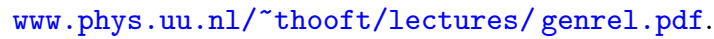

[40] D. N. Mermin, It's About Time: Understanding Einstein's Relativity, Princeton Univ. Press, Princeton, NJ, 2005.

[41] Б. М. Барбашов, В. В. Нестеренко, Модель релятивистской струны в физике адронов, Энергоатомиздат, М., 1987.

[42] H. Collins, Gravity's Shadow: The Search for Gravitational Waves, University of Chicago Press, Chicago, 2004.

[43] T. Damour, "General relativity and experiment", XIth International Congress on Mathematical Physics, ed. D. Iagolnitzes, International Press, Cambridge, MA, 1995, 37-46.

[44] B. Green, The Elegant Universe. Superstrings, Hidden Dimensions, and the Quest for the Ultimate Theory, Norton, New York, 1999.

Поступила в редакцию 20.08.2008 